EESTI NSV TEADUSTE AKADEEMIA TOIMETISED. 31. KOIDE FOOSIKA * MATEMAATIKA. 1982, NR. 1

ИЗВЕСТИЯ АКАДЕМНИ НАУК ЭСТОНСКОП ССР. ТОМ 31 ФИЗНКА * МАТЕМАТИКА. 1982, № 1

И. КЕИС

\title{
СУБОПТИМАЛЬНЫИ СИНТЕЗ УПРАВЛЕНИЯ МЕТОДОМ ИНВЕРСИИ И АГРЕГИРОВАНИЯ
}

\author{
(Представил Н. Алумяэ)
}

В работе, продолжающей исследования $\left[{ }^{1-6}\right]$, рассматривается субоптимальный синтез регулятора многомерной динамической системы с интегральным критерием качества и выпуклой компактной областью управления $\tilde{\Omega}$. Предложено два способа агрегации исходной $n$-мерной системы в $l$-мерную систему сравнения $(l \ll n)$ с информативной по $x, x^{(1)}$-управляемости вектор-функцией $y(t, \xi, c)$, где $c-$ векторный параметр агрегации. Первый (инверсный) способ задан производящим потенциалом $V^{+}(t, y, c)$ в случае существования максимина по $z, u$ гамильтониана системы. Второй способ определяется специальной внутренней нестационарной аппроксимацией $\Omega \subseteq \tilde{\Omega}$ области управления $\tilde{\Omega}$, дающей $t, y$-автономность системы. Получены субоптимальные регуляторы, оценки для оптимальной функции и условия минимизации показателя субоптимальности по вектору агрегацин $c$.

1. На основании результатов $\left[{ }^{1-5}\right]$ и их модификаций $\left[{ }^{6,7}\right]$ применим агрегацию к задаче оптимальной стабилизации движения $\xi \equiv 0$ по всем (некоторым) переменным динамической системы с интегральным показателем качества. Схема агрегации включает три этапа:

1. Введение информативных переменных-агрегатов $y_{k}=y_{k}(t, \xi, c)$ $(k=\overline{1, l}=\operatorname{dim} y \ll n)$, зависящих от постоянного вектор-параметра агрегации $c$.

2. Агрегирование исходной модели и показателя качества согласно рассматриваемому способу (первый уровень агрегирования). В результате для $\forall c \in \delta$ получаем агрегированную систему размерности $1+l \ll n$, для которой находим субоптимальный стабилизатор $u(t, \xi, c)$ исходной системы.

3. Поиск оптимального по критерию (мере) субоптимальности значения $c=c^{0}$, минимизирующего некоторую оценку погрешности (невязку) (второй уровень агрегирования).

Рассмотрим управляемую систему

$$
\begin{gathered}
\xi=F(t, \xi, u), R=\{t \geqslant 0,|x| \leqslant H\}, R_{0}=R \backslash Q, \\
Q=\{t, \xi \mid x(1)=0\}, \xi=\left[\begin{array}{l}
x \\
x^{(3)}
\end{array}\right] .
\end{gathered}
$$

$x=\left[\begin{array}{l}x^{(1)} \\ x^{(2)}\end{array}\right], \quad \operatorname{dim} x^{(i)}=n_{i}, \quad \sum_{i=1}^{3} n_{i}=n=\operatorname{dim} \xi, \quad \operatorname{dim} u=r, \quad u \in \tilde{\Omega}$,

$$
I[u \mid t, \xi]=\int_{t}^{t} F_{0}(\tau, \xi, u) d \tau \rightarrow \min _{u} \triangleq \nabla(t, \xi), \quad t \leqslant t_{1} \leqslant+\infty \quad\left(F_{0} \geqslant 0\right),
$$




$$
\begin{aligned}
& t_{1}=\min t^{*}: x^{(1)}[t] \rightarrow 0, \quad t \rightarrow t_{-}^{*}, \quad \tilde{\Omega}=\left\{u \mid \tilde{v}(u, \cdot) \leqslant v_{0}\right\} \quad\left(v_{0}=\text { const }>0\right), \\
& \tilde{v}=\tilde{v}(u, t, \xi), \quad \tilde{v}>0 u \neq 0, \quad \tilde{v}(\lambda u, \cdot)=|\lambda| \tilde{v}(u, \cdot) \quad(\forall|\lambda| \in[0, \infty)),
\end{aligned}
$$

где «коническая» функция $\tilde{v}(u, \cdot)$ строго выпукла по $u$ вне $u=\lambda e$ лучей: $\tilde{v}\left(u_{2}, \cdot\right)-\tilde{v}\left(u_{1}, \cdot\right)>\partial \tilde{v} / \partial u_{1} \cdot\left(u_{2}-u_{1}\right) \quad\left(\left(u_{2} \cdot u_{1}\right) \neq\left|u_{2}\right| \cdot\left|u_{1}\right|, \quad e=|u|^{-1} u\right.$, $|e|=1, \lambda=\operatorname{Re} \lambda)$. Смысл стабилизации регулятором $u(t, \xi, c)$ состоит в устойчивости по $x$ (при $\left.t_{1}=+\infty\right)$ и примыкании $x^{(1)}$-компоненты $\xi$ к цели $Q$ при $t \rightarrow t_{1}$ слева. Выберем $l \ll n$ независимых новых переменных $y_{k}(t, \xi, c)$ с учетом следующих требований: $y_{k}$ - функции лишь измеряе-

мых (наблюдаемых) величин $h_{s}(t, \xi), s=\overline{1, b}$, и вектора $c$. Агрегаты $y, y^{(1)}-$ информативные вектор-функции: $y \rightarrow 0 \Rightarrow x \rightarrow 0, y^{(1)} \rightarrow 0 \Rightarrow$ $\Rightarrow x^{(1)} \rightarrow 0 \quad(\forall c \in \delta, \quad \operatorname{dim} c=m) \quad$ равномерно по $t, z \in R_{0}$. Пусть $y \in C(R \times \delta), y \in C_{1}\left(R_{0} \times \delta\right)$, а $y \equiv 0$ при $x=0, y^{(1)} \equiv 0$ при $x^{(1)}=0$. Областью изменения параметра агрегации $c=\left(c_{1}, \ldots, c_{m}\right)^{*}$ считаем открытый шар $\delta(0, \varrho), \varrho=$ const $\leqslant \infty, y^{(1)}=\left(y_{1}, \ldots, y_{d}\right)^{*}, d \leqslant l \ll n$. В силу $\operatorname{det}\left[\partial y_{k} / \partial x_{\sigma}\right] \neq 0$ на $R_{0}(k, \sigma=\overline{1, l})$ из (1.1) с учетом свойств $y(t, \xi, c)$ имеем эквивалентную систему и задачу оптимальной $y, y^{(1)}$-стабилизации $q \equiv 0$ (при $u \equiv 0$ ) в переменных $y, z$ :

$$
\begin{gathered}
y=Y(t, q, u, c), \quad z=Z(\cdot), q \in D_{0}=q\left(R_{0}\right), q=\left[\begin{array}{l}
y \\
z
\end{array}\right], \quad z=\left[\begin{array}{l}
x_{l+1} \\
x_{n}
\end{array}\right], \\
I=\int_{t}^{t_{1}} f_{0} d \tau \rightarrow \underset{u}{\min \triangleq V}=V, \quad f_{0}=\left.F_{0}\right|_{\xi \rightarrow q}, \quad v=\left.\tilde{v}\right|_{\xi \rightarrow q}, \quad q=(d q / d t)_{\xi \rightarrow q}, \\
u \in \Omega=\left\{v(u, \cdot) \leqslant v_{0}\right\}, \quad v(u, t, q, c)=v(u, \cdot) \in\{\tilde{v}(u, \odot)\} .
\end{gathered}
$$

Обозначим множества $y, y^{(1)}$ - и $x, x^{(1)}$-стабилизаторов систем $(1.1)$ и (1.2) с соответствующими областями стабилизации $\Gamma(c)=$ $=\left\{0 \leqslant t_{0} \leqslant T_{0}(c),\left|y_{0}\right| \leqslant \eta_{0}(c),\left|z_{0}\right| \leqslant \gamma_{0}(c)\right\}$ и $\tilde{\Gamma}(c)=\left\{\cdot\left|x_{0}\right| \leqslant \tilde{\eta}_{0}(c), \cdot\right\}$ $(\Gamma \rightarrow \tilde{\Gamma})$ через $U=\{u(t, q, c)\}$ и $\tilde{\sigma}=\{\tilde{u}(t, \xi, c)\}$. Стабилизирующий по $y, y^{(1)}$ регулятор $u(t, q, c)$ системы (1.2) будет $x, x^{(1)}$-стабилизатором $\widetilde{u}(t, \xi, c)$ системы (1.1) в силу информативности $y, y^{(1)}$. Ниже будем считать, что вектор-функция $u(t, q, c)$ удовлетворяет одному из критериев $y, y^{(1)}$-стабилизируемости $\left[\beta^{3,4,8-11}\right]$, задающих постоянные $T_{0}, \eta_{0}, \gamma_{0}-$ размеры области стабилизируемости $\Gamma(c) \rightarrow \tilde{\Gamma}(c)$.

2. Инверсный способ - обращение задачи с производящим потенциалом

$$
\begin{gathered}
V^{+}=V^{+}(t, y, c) \underset{D_{0}}{>0},\left.V^{+}\right|_{\substack{(1) \\
y=0}} \equiv 0, V^{+} \in C(D), V^{+} \in C_{1}\left(D_{0}\right) \\
\forall c \in \delta(D=q(R)) .
\end{gathered}
$$

Примем, что $V^{+}$и $(1.2)$ удовлетворяют двум условиям. Во-первых, существует седловая точка $z^{+}(t, y, c), u^{+}(t, y, c) \in \Omega \cap \Omega^{+}, \quad u^{+} \in C\left(D_{0}\right)$ для $\forall t, y \in D_{0}, c \in \delta$ гамильтониана $H^{+}(z, u, \cdot)$ системы (1.2) при $V \rightarrow V^{+}$, т. е. выполнены неравенства

$$
\begin{gathered}
H^{+}\left(z^{+}, u, \cdot\right) \geqslant \max _{z} \min _{u} H^{+}=H^{+}\left(z^{+}, u^{+}, \cdot\right) \triangleq-h_{+}(t, y, c) \geqslant H^{+}\left(z, u^{+}\right) \\
\quad\left(u \in \Omega, q \in D_{0}\right), \\
H^{+}\left(z^{+}, u, \cdot\right) \geqslant H^{+}\left(z^{+}, u^{+}, \cdot\right) \triangleq-h_{+}\left(H^{+}=H\left[V^{+}, z, u, \cdot\right] \triangleq\right. \\
\left.\triangleq V_{t}^{+}+Y \cdot V_{y}^{+}+f_{0}, f_{x} \triangleq \partial \partial f / \partial x\right),
\end{gathered}
$$




$$
\begin{gathered}
\left(z^{+}(t, y, c) \in C_{1}\left(D_{0}\right) \quad \forall c \in \delta, \Omega^{+} \triangleq\left\{u|v|_{z=z^{+}} \leqslant v_{0}\right\}\right), \\
H^{+}\left(z, u^{+}, \cdot\right) \leqslant H^{+}\left(z^{+}, u^{+}, \cdot\right) \equiv-h_{+}(t, y, c) .
\end{gathered}
$$

Во-вторых, вектор-функция $u^{+}(t, y, c)$ - стабилизатор (1.2) $\left(u^{+} \in U\right)$ на $\Gamma(c) \in D_{0}(c)$, где все рассматриваемые функции имеют необходимую гладкость.

Рассмотрим вспомогательную задачу оптимальной стабилизации заданием агрегированной из (1.2) системы вида

$$
\begin{gathered}
y_{+}^{*}=\left.Y\right|_{z=z^{+}}, \quad I_{+}=\int_{t}^{t_{+}} f_{+} d \tau \rightarrow \min _{u} \triangleq V_{+} \quad\left(t_{+} \neq t_{1}, z^{+} \in C_{1}\left(D_{0}\right)\right), \\
\left.f_{+} \triangleq f_{0}\right|_{z=z^{+}}+h_{+},\left.\quad u \in \Omega^{+} \triangleq \Omega\right|_{z=2^{+}} .
\end{gathered}
$$

Пусть $u^{+}(t, y, c) \in U^{\prime \prime}$, т. е. $u^{+}-y, y^{(1)}$-стабилизатор (2.3) при $q \in \Gamma(c)$. Обозначим гамильтониан агрегированной системы (2.3) через $H_{+}=$ $=H^{+}\left(z^{+}, u, \cdot\right)+h_{+}$, допуская $V_{+} \equiv V^{+}$. Интегрируя (2.1) вдоль (2.3) при $\forall u \in U^{\prime \prime}$ и начальных значениях $t_{0}, y_{0}$, из $\Gamma(c)$ с учетом $\left.H_{+}\right|_{u=u^{*}} \equiv$ $\equiv 0$ находим $V_{+}=V^{+}=\min I_{+}=I_{+}\left[u^{+}\right] \leqslant I_{+}[u]$. Таким обра-

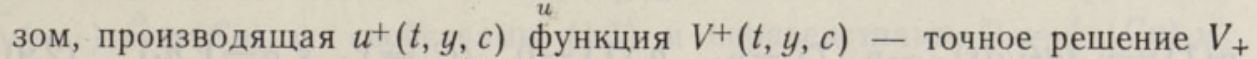
вспомогательной задачи (2.3) согласно определению субоптимального решения.

Найдем оценку сверху субоптимальной функции $I^{+} \triangleq I\left[u^{+}\right]$. Интегрируя (2.2) вдоль (1.2) при $u=u^{+}, t_{0}, q_{0} \in \Gamma(c)$, с учетом $u^{+} \in U$ находим неравенства

$$
\begin{gathered}
\int_{t_{0}}^{t^{+}}\left[\left(V^{+}\right) \cdot-H^{+}\left(z^{+}, u^{+}, \cdot\right)\right] d \tau \leqslant-\int_{t_{0}}^{t^{+}} f_{0}\left(\cdot, u^{+}, c\right) d \tau \quad\left(V^{+}\left[t^{+}\right]=0\right), \\
-\int_{t_{0}}^{t^{+}}\left[h_{+}+\left(V^{+}\right) \cdot\right] d \tau \geqslant \int_{t_{0}}^{t^{+}} f_{0}\left(\cdot, u^{+}, c\right) d \tau, \quad V^{+}\left[t_{0}\right] \geqslant \int_{t_{0}}^{t^{+}}\left[f_{0}\left(\cdot, u^{+}, c\right)+h_{+}\right] d \tau, \\
I_{+} \triangleq I\left[u^{+}\right] \leqslant V^{+}-\int_{t_{0}}^{t^{+}} h_{+} d \tau=\int_{t_{0}}^{t^{+}}\left(\left.f_{0}\right|_{z=z^{+}, u=u^{+}}\right) d \tau .
\end{gathered}
$$

Если $h_{+} \geqslant 0$, то из (2.4) следует $I^{+} \leqslant V^{+}$или $I^{+} \leqslant \nabla^{+}$в переменных $\xi$. Заметим, что введение операции $\max$ не только соответствует «игре против природы», но и дает искомую $\underset{t}{t}, y$-автономность субоптимального решения $V^{+}, u^{+}$.

Для поиска наилучшего вектора $c$ на втором уровне агрегации замкнем систему (1.1) субоптимальным регулятором $u_{+}(t, \xi, c)=$ $=\left.u^{+}\right|_{q \rightarrow \xi}$. Получим модель

$$
\begin{array}{cc}
\xi=\Phi(t, \xi, c), \quad c^{*}=0, \quad x^{(1)}[T]=0 \quad\left(T \triangleq t^{+}, u_{+} \in \mathscr{U}, \operatorname{dim} x^{(1)}=n_{1}\right), \\
\xi[t] \in C\left[t_{0}, T\right], \quad \xi_{0} \triangleq \xi\left[t_{0}\right],\left.\quad \Phi \triangleq F\right|_{u=u_{+} .}
\end{array}
$$

Примем, что пересечение $\bigcap_{c \in \delta} \tilde{\Gamma}(c) \triangleq \tilde{\Gamma_{0}}=\left\{0 \leqslant t_{0} \leqslant \tilde{T}_{*},\left|x_{0}\right| \leqslant \tilde{\eta_{*}},\left|x_{0}^{(3)}\right| \leqslant \tilde{\gamma_{*}}\right\}$ непусто. Пусть $t_{0}, \xi_{0} \in \tilde{\Gamma}_{0}$. Поставим задачу минимизации по $c$ точечного критерия субоптимальности вида

$$
\tau^{+}=\int_{t_{0}}^{t^{*}} \Phi_{0}(\tau, \xi, c) d \tau \triangleq T\left[u_{+}\right] \quad\left(t^{+}=T[c],\left.\Phi_{0} \triangleq F_{0}\right|_{u=u_{+}}\right)
$$

при дифференциальных и краевых условиях (2.5) со свободным $T$. Для 
ее автономизации введем эквивалентную расширенную $\left(x_{n+1} \equiv t\right)$ систему

$$
x_{0}=\Phi_{0}, \quad \xi=\Phi, \quad x_{n+1}=1, \quad c=0 \quad\left(x^{(1)}[T]=0\right)
$$

и уравнения сопряженных переменных (импульсов)

$$
\begin{gathered}
p_{0}^{*}=-\partial H / \partial x_{0}=0, \quad p=-\partial H / \partial \xi=-\partial G / \partial \xi, \\
p_{n+1}^{\cdot}=-\partial H / \partial x_{n+1}=-\partial G / \partial x_{n+1}, \quad p_{0}=\text { const, } \\
H=G+p_{n+1}+0 \cdot \psi, \quad G=p_{0} \Phi_{0}+p \cdot \Phi, \quad \operatorname{dim} \psi=\operatorname{dim} c=m .
\end{gathered}
$$

Из (2.7), (2.8) и условий трансверсальности $\left[{ }^{12,13}\right]$ имеем $n+n_{1}+m+1$ уравнений

$$
\begin{gathered}
\left(t_{0} \leqslant t \leqslant T\right) \\
-\left.v_{s} \frac{\partial G_{s}}{\partial t}\right|_{T}=H[T]=H(t)=0 \sim p_{n+1}+G=0, \\
p_{n+1}(T)=\left.v_{s} \frac{\partial G_{s}}{\partial x_{n+1}}\right|_{T}=0 \sim G(T)=0, \\
p(T)=v_{\sigma}(\partial G / \partial x)_{T} \sim p_{\sigma}(T)=v_{\sigma}, \quad p_{r}(T)=0 \quad\left(G_{\sigma} \equiv x_{\sigma}, \quad s, \sigma=\overline{1, n_{1}}\right), \\
\psi\left(t_{0}\right)=0, \quad \psi(T)=\int_{t_{0}}^{T}(\partial H / \partial c) d \tau=\int_{t_{0}}^{T}(\partial G / \partial c) d \tau=0 \quad\left(r=\overline{n_{1}+1, n}\right)
\end{gathered}
$$

для определения $n+n_{1}+m+2$ неизвестных: импульсов при $t=T$, времени $T$ и постоянных $c, p_{0}, v_{\sigma}\left(p_{0}^{2}+\sum_{\sigma=1}^{n_{1}} v_{\sigma}^{2} \neq 0\right)$, где последние заданы с точностью до множителя, $p_{0} \leqslant 0$, причем $p_{0}=-1$ в нормальном случае. Численное решение уравнений $(2.7)-(2.9)$ дает значение оптимального вектора агрегации $c=c^{+}=c^{+}(t, \xi)$ и уточненную оценку сверху оптимальной функции $\widetilde{\nabla} \leqslant T^{+}\left(t, \xi, c^{+}\right) \leqslant I^{+}(t, \xi, c)$ в предположении существования и единственности их решения. Однако из-за необходимости интегрировать $2 n+m$ уравнений $(2.7)-(2.9)$ и знать $z[t]$ вычисление $\tilde{I}^{+}$-оптимального $c$ для каждой точки из $\tilde{\Gamma}_{0}$ превращается в сложную процедуру. Поэтому имеет смысл искать для (2.6) и (2.7) наилучший $c_{(s)}+$ по $I_{(s)}$-объемному критерию субоптимальности

$$
I_{(s)}^{s}=\int_{\tilde{\Gamma}_{0}}\left(\tilde{I}^{+}\right)^{s} d \omega, \quad I_{\infty}=\max _{\tilde{\Gamma}_{0}} T^{+}, \quad c_{s}^{+}=\operatorname{opt} c\left[I_{(s)}\right] \quad(s=1,2, \ldots, \infty) .
$$

Из необходимых условий вариационного исчисления для поиска $c_{(s)}{ }^{+}-$ наилучшего по (2.10) значения - в неособенных случаях находим оптимальный параметр. Процесс поиска $z^{+}, u^{+}$упрощается, если $z$-компонента $\xi$ состоит из угловых переменных $\varphi_{\mu} \leqslant 2 \pi, \mu=\overline{l+1, n}$.

3. Способ агрегации внутренней (нестационарной) аппроксимацией $\Omega$ исходной области управления $\tilde{\Omega}=\left\{|u| \leqslant Q^{0}\right\}, \varrho^{0}=$ const $>0$. Для простоты ограничимся (в качестве примера) применением агрегирования к задаче оптимальной $x, x^{(1)}$-стабилизации, линейной по $u$ системы с ядром интеграла качества, зависящим в новых переменных лишь от $t, y, c$

$$
\begin{gathered}
\xi=X(t, \xi)+A(t, \xi) u, \quad R=\{t \geqslant 0,|x| \leqslant H\}, \quad R_{0}=R \backslash x^{(1)}=0, \\
I=\int_{t}^{t_{1}} f_{0}(\tau, y, c) d \tau \rightarrow \min _{u} \triangleq \tilde{S}, \quad u \in \tilde{\Omega}=\left\{|u| \leqslant \mathrm{Q}^{0}\right\}, \quad \mathrm{Q}^{0}=\text { const }>0 .
\end{gathered}
$$


Примем, что система (3.1) имеет известные $x, x^{(1)}$-информативные агрегаты $y_{k}(t, \xi, c) \quad(k=\overline{1, l} \ll n)$, которые вместе с (3.1) удовлетворяют условиям

$$
\left.y\right|_{u=0}=Y(t, y, c) \Rightarrow y=Y+B^{*} u \quad\left(B=A^{*}(\partial y / \partial \xi)^{*}=\left[\begin{array}{l}
B_{1} \\
B_{2}
\end{array}\right]\right),
$$

$\operatorname{rank} B_{1}=l \triangleq \operatorname{dim} y \leqslant \operatorname{dim} u \triangleq r ; \quad \inf _{R_{0}} \lambda_{+}>0, \quad \sup _{R_{0}} \lambda^{+}<\infty \quad(\forall c \in \delta)$,

где $0<\lambda_{+}, \lambda^{+}-$соответственно наименьший и наибольший корни уравнения

$\operatorname{det}\left[C^{*} C-\lambda^{2} 1_{r}^{r}\right]=0, \quad|C| \neq 0, \quad C \triangleq\left[\begin{array}{cc}0_{l}^{r-l} \\ B & 1_{r-l}^{r-l}\end{array}\right]-$ матрица $(r \times r)$.

Внутреннюю нестационарную (зависящую от $t$, $)$ аппроксимацию $\Omega$ области $\tilde{\Omega}$ зададим симметрической «конической» функцией $\tilde{v}(u, \cdot) \triangleq v(C u)$ со свойствами (1.1) и неравенствами $\Omega=\{v(C u) \leqslant$ $\leqslant v_{0}=$ const $\left.>0\right\}$, где $v_{0}=\varrho^{0} \mu_{0}, \mu_{0}=\min v \cdot \inf _{R_{0}} \lambda_{+}>0, e=u /|u|,|e|=1$, $\mu^{0}=\max _{e} v \cdot \sup _{B_{0}} \lambda^{+}<\infty$. В силу (3.2) граница $\left\{u^{\prime}\right\}$ области $\Omega$ лежит в кольце $\varrho_{0} \leqslant\left|u^{\prime}\right| \leqslant \mathrm{Q}^{0}, \operatorname{dim} \Omega=r, \quad \varrho_{0}=$ const $>0$, причем $\varrho_{0}=$ $=\varrho^{0} \mu_{0} / \mu^{0}$.

При таком специальном выборе аппроксимации $\Omega \subseteq \tilde{\Omega}$ агрегирование $(3.1)$ с учетом (3.2) исчерпывается заменой $\tilde{\Omega}$ на $\Omega\left(t_{1} \not \equiv t^{\prime} !\right)$ и дает систему

$$
\begin{gathered}
y=Y(t, y, c)+B^{*}(t, \xi, c) u, \quad I^{\prime}=\int_{t}^{t^{\prime}} f_{0} d \tau \rightarrow \min _{u} \Rightarrow S(t, y, c) \\
\left(u \in \Omega, t_{1} \rightarrow t^{\prime}, I \rightarrow I^{\prime}\right)
\end{gathered}
$$

с гамильтонианом, линейным по $u$ на строго выпуклом $\Omega$-компакте

$$
H^{\prime}=S_{t}+p \cdot Y+C^{*} \psi \cdot u \quad\left(p \triangleq S_{y}, \psi=(p, 0)^{*}, \operatorname{dim} \psi=\operatorname{dim} u=r\right) .
$$

Введем «коническую» функцию $W(u) \leftrightarrow v(v)$ равенством

$$
W(u)=\max _{v(v)=1}(u \cdot v) \in\{v(u, \cdot)\}\left(\max _{e}^{-1} v \leqslant|u|^{-1} W \leqslant \min _{e}^{-1} v, e=|u|^{-1} u\right) .
$$

Из (3.3), (3.4) и конструкции $\tilde{v}(u, \cdot) \equiv v(C u)$ следует, что уравнение Беллмана-Якоби для оптимальной функции $S$ задачи (3.3) $t, y$-автономно и имеет размерность $l+1 \ll n$

$$
\begin{gathered}
S_{t}+p \cdot Y-v_{0} w(p)+f_{0}=0 \\
\left(\left.S\right|_{y^{(1)}=0} \equiv 0,\left.\frac{\partial S}{\partial y_{\alpha}}\right|_{y^{(1)=0}}=0, w(p) \equiv W(\psi), \alpha=\overline{d+1, n}\right) .
\end{gathered}
$$

Субоптимальный регулятор системы (3.1)-(3.2), оптимальный для ее агрегированной формы (3.3), в общем случае зависит от всех переменных $t$, , причем является граничным для области $\Omega$ и потенциальным по структуре 


$$
u^{\prime}=u^{\prime}(t, \xi, c)=-v_{0} C^{-1} W_{\psi} .
$$

Вид $u^{\prime}$ определен в (3.7) решением уравнения (3.6) при условии $y, y^{(1)}$-стабилизации $(3.3)$, что эквивалентно здесь $x, x^{(1)}$-стабилизации (3.1).

Используя формулы (2.5)-(2.10), можно аналогично предыдущему искать наилучший векторный параметр агрегации $c=c^{0}$ по точечному или объемному критерию субоптимальности. Замыкая (3.1) ее стабилизатором $\tilde{u}^{\prime}\left(t, \xi, c^{0}\right)$, заданным (3.6) и (2.7), при этих критериях субоптимальности найдем для оптимальной функции $\widetilde{S}$ задачи (3.1) оценки сверху $\tilde{S} \leqslant S\left(t, y, c^{0}\right), \widetilde{S} \leqslant I\left[u^{\prime}\left(\cdot, c^{0}\right)\right]\left(\forall c^{0} \in \delta\right)$.

В заключение отметим, что агрегирование системы (3.1) при условиях (3.2) сводится лишь к выбору специальной аппроксимации $\Omega$ области управления $\tilde{\Omega}$ при сохранении исходной модели.

\section{Л ИТЕРА Т У РА}

1. Aoki, M., In: Optimization Methods for Large-Scale Systems, 5 Aggregation, McGraw Hill Book Company, New York, 1971, p. 191-232.

2. У л ь м С. Ю., Автоматика и телемеханика, № 5, 27-32 (1972).

3. З емляков А. С., М атр осов В. М., В кн.: Оптимальное и адаптивное управление, Саратов, Сарат. ун-т, 1977 , с. $202-215$.

4. Grujič, Lj. T., Gentina, I. C., Borne, P., Int. J. Control, 24, № 4, 529-537 (1976).

5. Первозв анский А. А., Гайцгори В. Г., Декомпозиция, агрегирование и приближенная оптимизация, М., «Наука», 1979, с. $154-244$.

6. Ке й с И., Изв. АН ЭССР, Физ. Матем., 26, № 3, 243-251 (1977).

7. Ке й с И., Изв. АН ЭССР, Физ. Матем., 28, № 2, 107-114 (1979).

8. К ра сов в ки й Н. Н., В кн.: Малкин И. Г. «Теория устойчивости движения», доп. 4, М., «Наука», 1966, с. $475-514$.

9. Р у м я нц е в В. В., Прикл. мат. и мех., 34, вып. 3, 440-456 (1970).

10. Дем ин В. Г., Фура сов В. Д., Прикл. мат. и мех., 40, вып. 2, 355-359 (1976).

11. Ке й с И., Изв. АН ЭССР, Физ. Матем., 27, № 3, 274-288 (1978).

12. Мои сее в Н. Н., Чнсленные методы в теории оптимальных систем, М., «Наука», 1971 , c. $32-34$.

13. Болтянский В. Г., Математические методы оптимального управления, М., «Наука», 1966, с. 295-300.

Институт кибернетики

Академии наук Эстонской ССР

Поступила в редакцию $17 / \mathrm{VI} 1981$

\section{KEIS}

\section{DUNAAMILISTE SUSTEEMIDE REGULAATORITE SUBOPTIMAALNE SUNTEES POORD- JA AGREGEERIMISMEETODIL}

Artiklis on vaadeldud mittelineaarse dünaamilise süsteemi juhtimise ülesannet integraalse efektiivsusindeksi korral. Optimaalse sünteesi ülesande ligikaudseks lahendamiseks on esitatud kaks $l \ll n$-mõõtmelise süsteemi agregeerimisviisi. Neist esimesel on etteantud $V^{+}(t, y, c)$ potentsiaal ja tingimused $(2.1),(2.2)$, teine lahendusviis baseerub spetsiaalsel kumera kompakti $\tilde{\Omega} \rightarrow \Omega$ teisendusel. On leitud suboptimaalsed regulaatorid $u_{+}, u^{\prime}$, funktsionaali $I\left[u_{+}\right]$hinnangud $(2.4)$ ning optimaalse agregeerimisvektori $c$ määramise tingimused. 


\section{KEIS}

\section{THE SUBOPTIMAL CONTROL SYNTHESIS VIA INVERSION AND AGGREGATION}

IN MULTIDIMENSIONAL DYNAMICAL SYSTEMS

The optimal $x, x^{(1)}$-stabilization problem for large-scale $n$-dimensional nonlinear dynamical system is considered. For convenience the convex-compact domain of controls $\{u\}=\tilde{\Omega}$ is described by one conical function $\tilde{v}(u, \cdot)$. The principal scheme of the approach includes three following stages.

1. The introduction of the informative variables $y_{k}=y_{k}(t, \xi, c), k=\overline{1, l} \ll n$ as the aggregates of the state-vector $\xi=\left(x^{*}, x^{(3) *}\right)^{*}$, where $\delta \ni c-$ the constant aggregation vector.

2. The aggregation procedure covering both the model (1.1) and its performance index, respectively.

3. The optimal for the prescribed criterion $c$-choice.

Two new methods of aggregation are suggested in the paper. When $u^{+} \in U \cap U^{\prime \prime}$, the first one is valid under the maxmin conditions $(2.1),(2.2)$ on the potential $V^{+}(t, y, c)$ and the system (1.2). Hence the suboptimal control $u_{+}$and the inequalities (2.4) for the suboptimal function $I\left[u^{+}\right]$are obtained. Necessary conditions for the choice of the vector $c$ minimizing the criterion (2.6) are derived from (2.7) $-(2.9)$.

The second method of aggregation is based on the special approximation of the initial domain $\tilde{\Omega}=\left\{|u| \leqslant \mathrm{Q}^{0}\right\}$ for the linear in $u$ system (3.1) with (3.2) properties. As a result, the suboptimal control (3.7) and $l+1$-dimensional Bellman-Jacoby equation (3.6) are obtained. 